\title{
Informatik akfuell
}

F. Huber-Wäschle H. Schaver P. Widmayer (Hrsg.)

GISI 95

Herausforderungen eines globalen Informationsverbundes für die Informatik 


\title{
WULPUS \\ Wissensbasierte Hilfen und Erklärungen für ein betriebswirtschaftliches Planspiel
}

\author{
Claus Möbus, Olaf Schröder, Heinz-Jürgen Thole* \\ Universität Oldenburg, Fachbereich Informatik, \\ Abteilung Lehr- / Lernsysteme, D-26111 Oldenburg, und \\ OFFIS - Oldenburger Forschungs- und Entwicklungsinstitut \\ für Informatik-Werkzeuge und -Systeme, D-26121 Oldenburg \\ \{moebus, schroeder, thole\}@informatik.uni-oldenburg.de
}

\section{Einleitung}

In der betriebswirtschaftlichen Aus- und Weiterbildung werden in zunehmenden Maße Planspiele eingesetzt, um dem steigenden Bildungsbedarf kostengünstig zu begegnen. EDVgestützte Simulationen und Planspiele haben durch die forcierte Vermittlung von Schlüsselqualifikationen eine neue Bedeutung erlangt. Man erhofft sich dadurch einerseits die Vertiefung der Analyse- und Problemlösefähigkeiten und andererseits die Verbesserung der Teamfähigkeit.

Grundlage eines Planspiels bildet ein Modell des Realitätsausschnitts (z.B. Schoel, 1988). Für umfangreiche Problemstellungen, wie z.B. die Führung eines Unternehmens, das sich gegenüber den Mitbewerbern auf den Absatzmärkten zu behaupten hat, muß im Planspiel stärker von der Realität abstrahiert werden (Ebert, 1978; Eser 1992). Dabei bildet die damit verbundene Komplexität des Modells einen Kompromiß zwischen den Extremen:

- große Komplexität, um Realitätsnähe zu garantieren

- geringe Komplexität, um Zusammenhänge transparent werden zu lassen.

Damit Modelle komplexer betriebswirtschaftlicher Gegenstandsbereiche nicht zu stark von der Realität abweichen, weisen sie einen hohen Vernetzungsgrad ihrer Bestandteile auf. Dadurch entsteht zwangsläufig Intransparenz. Der Zusammenhang zwischen Entscheidungs- und Ergebnisvariablen eines Unternehmens wird durch weitere Komponenten wie z.B. das Verhalten der Mitbewerber beeinflußt. In herkömmlichen Planspielen sind die Entscheidungen unabhängige und die Ergebnisse abhängige Variablen. Für den Benutzer ist der Zusammenhang zwischen Entscheidungen und den resultierenden Ergebnissen häufig schwer nachvollziehbar, falls das Domänenwissen des im Planspiel modellierten Weltausschnitts fehlt oder die im Modell enthaltenen Abstraktionen der Realität subjektiv stark verfremdend wirken (Eser, 1992; Ochs \& Steinmann, 1976). Eine weitere Quelle von Schwierigkeiten für den Benutzer ist die Identifikation zeitverzögerter Effekte.

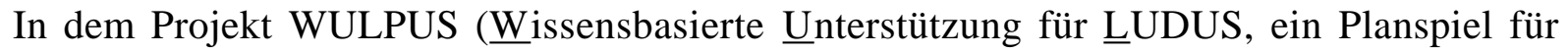
Unternehmensstrategien) wurde ein Ansatz entwickelt, der aufzeigt, wie die Zusammenhänge zwischen verschiedenen betriebswirtschaftlichen Teilbereichen durch einen Hypothesentestansatz (Möbus \& Thole, 1990; Möbus, Thole \& Schröder, 1993) sowie durch wissensbasierte Erklärungen für den Lernenden transparent gemacht werden können. Die Fähigkeit, Hypothesen zu formulieren und zu überprüfen, ist eine Schlüsselqualifikation. Explizites, selbstständiges Formulieren von Hypothesen hat einen effizienzsteigernden und optimierenden Einfluß auf die Wissensakquisition bei den Lernenden (Shute \& Glaser, 1990). Die betriebswirtschaftliche Domäne von WULPUS ist dabei beschränkt auf einen Teilbereich der Inhalte des klassischen Planspiels LUDUS der Firma rado-plan.

\footnotetext{
*Wir danken Klaus Adam und Hagen von Stuckrad für die Mitwirkung an der Implementation.
} 
Zentrale Unterscheidungsmerkmale zwischen WULPUS und LUDUS sind:

- Hypothesentesten vor dem "echten Spielschritt": Der Lernende kann sich zum Beispiel die Konsequenzen verschiedener Entscheidungskombinationen berechnen und anzeigen lassen. Im Unterschied zu LUDUS können alternative Entscheidungskombinationen "durchgespielt" werden, sei es, um die Beziehungen zwischen den Variablen zu studieren oder um vor der eigentlichen Entscheidungsfindung Hypothesen über eine günstige Kombination der Entscheidungsvariablen zu formulieren und zu überprüfen.

- Vorwärts- vs. Rückwärtsplanung: Neben der Eingabe von Entscheidungen kann sich der Benutzer Ziele (im Sinne gewünschter Ergebnisse) setzen. Das System versucht dann, diese Hypothese über die Erreichbarkeit der Ziele zu vervollständigen, indem es mit den gewünschten Ergebnissen konsistente Entscheidungskombinationen vorschlägt.

- Korrekturmöglichkeit von inkonsistenten Entscheidungs- und Ergebnisvariablen: Der Benutzer wird auf nicht erreichbare Ziele hingewiesen und erhält vom System für die betreffenden Variablen Korrekturvorschläge.

- Erklärungskomponente: Der Benutzer erhält wissensbasierte Erklärungen für:

(a) das Zustandekommen der Ergebnisse bei den getroffenen Entscheidungen,

(b) die vom System vorgeschlagenen Entscheidungen zur Erreichung seiner gesetzten Ziele,

(c) die Nichterfüllbarkeit der gesetzten Ziele.

Für die Übertragung des Hypothesentestansatzes auf ein Planspiel sind der Rollentausch der Entscheidungs- und Ergebnisvariablen, das Aufschieben von Berechnungen, bis die benötigten Werte vorliegen, und die Korrekturmöglichkeit von Inkonsistenzen notwendige Voraussetzungen. Da LUDUS diese Einrichtungen nicht bietet, war es nicht möglich, eine Komponente zu entwickeln, die lediglich via Schnittstellen mit LUDUS kommuniziert. Deshalb mußte der Planspielausschnitt vollständig rational rekonstruiert und neu programmiert werden.

\section{Kurzbeschreibung von LUDUS}

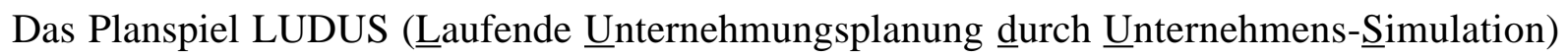
gehört zu der Gruppe der strategischen Konkurrenz-Unternehmensführungsspiele. Es wurde 1990 mit dem deutschen Hochschul-Software-Preis ausgezeichnet. Eingesetzt wird es vornehmlich an Hochschulen, aber auch in Industrie- und Beratungsunternehmen.

In dem Planspiel konkurrieren wahlweise 2 bis 5 Unternehmen mit 1 bis 3 Produkten auf 2 bis 4 Märkten. Die Planungsschwerpunkte liegen im Absatz-, Produktions-, Transport-, Personalund Finanzbereich, wobei die Entscheidungen in jedem dieser betrieblichen Teilbereiche die Auswirkungen der Entscheidungen in den anderen Teilbereichen beeinflussen.

Die Spielteilnehmer von LUDUS müssen ihre Entscheidungen vollständig abgeben. Diese können allerdings in beliebiger Reihenfolge eingegeben werden. Wenn alle Spieler (Unternehmen) ihre Entscheidungsfindung abgeschlossen haben, wird der Spielschritt durchgeführt, d.h. die Ergebnisvariablen werden berechnet. Die Spieler können sich die Auswirkungen ihrer Entscheidungen in Form des Berichtswesens anschauen und ihre Entscheidungen für die neue Periode treffen. Der Benutzer erhält keine Rückmeldung in Form 
von Begründungen über das Zustandekommen der Ergebnisvariablen und hat deshalb u.U. Probleme, seine neuen Entscheidungen den gewünschten Zielen anzupassen.

Als einzige Hilfe zur Entscheidungsfindung stellt LUDUS sogenannte "Planungshilfen" zur Verfügung. Der Spieler hat die Möglichkeit, ein Intervall für den geplanten Absatz und die Produktion anzugeben. Daraufhin berechnet das System z.B. die zum Transport auf weitere Märkte zur Verfügung stehende Menge von Produkten. Der Spieler wird nicht gewarnt, wenn die vorhandene Maschinenkapazität nicht ausreicht, um die geplante Produktion zu realisieren. Außerdem wird die Erreichbarkeit des geplanten Absatzes auf dem jeweiligen Markt vom System nicht geprüft, d.h. das Marktvolumen, die Maßnahmen der konkurrierenden Mitbewerber und das eigene Marketingmix werden nicht berücksichtigt.

LUDUS stellt den Benutzer daher vor folgende Probleme:

1. Es ist nicht deutlich, wie und warum die Ergebnisse aufgrund der getroffenen Entscheidungen zustandegekommen sind (Intransparenz, fehlende Erklärungen).

2. Probehandlungen sind nicht möglich. Entscheidungen können nicht rückgängig gemacht, Alternativen nicht einander gegenübergestellt werden.

3. Man kann sich keine Ziele setzen, und es bleibt unklar, mit welchen Entscheidungen die intendierten Ziele erreichbar sind.

\section{Beschreibung von WULPUS}

Das wissensbasierte Planspiel WULPUS wurde entwickelt, um einen Ansatz aufzuzeigen, der dem Lernenden die Zusammenhänge in komplexen Planspielen transparent macht.

WULPUS bietet dem Benutzer daher die folgenden Möglichkeiten:

1. Erklärungen anzufordern, um die Zusammenhänge zwischen Entscheidungen und Ergebnissen transparent zu machen

2. Probehandlungen durchzuführen und die resultierenden Ergebnisse gegenüberzustellen

3. Ziele zu setzen, also gewünschte Ergebnisse bzw. Teilergebnisse vorzugeben

Der WULPUS-Spieler wird in mehrfacher Hinsicht bei seiner Entscheidungsfindung unterstützt. Dazu wurde das Konzept einer "Simulation in der Simulation" entworfen und realisiert. Der Spieler hat die Möglichkeit, vor dem "echten Spielschritt" Alternativen zu testen, indem er das System wieder zurücksetzt und mit neuen Werten startet. Er kann so mehrere Perioden durchspielen, um zeitlich verzögerte Effekte zu erkennen. Außerdem kann er zusätzlich auf Wunsch die möglichen Mitbewerberentscheidungen variieren und damit das Verhalten der Konkurrenz simulieren, bzw. realistische Hypothesen über deren Verhalten aufstellen und vom System prüfen lassen. WULPUS unterstützt den Planspieler außerdem mit Erklärungen über die Nichterreichbarkeit von Zielen und über den Zusammenhang von Entscheidungs- und Ergebnisvariablen mit Hilfe einer integrierten Expertenwissensbasis. Es hat sich gezeigt, daß Lernende gerade bei komplexen Planspielen oft Probleme haben, Entscheidungen zu treffen, da sie die Auswirkungen nicht überschauen können. Ein Grund dafür ist das fehlende Vorwissen. Daraus folgt, daß Novizen einen geringen Wissenszuwachs beim Arbeiten mit einem komplexen Planspiel haben (vergl. Ripper et al., 1993). 
Sie haben jedoch genaue Vorstellungen über die Ergebnisse, die sie mit den zu treffenden Entscheidungen erreichen wollen. Deshalb haben wir das Planspiel WULPUS so konzipiert, daß ein Spieler zur Entscheidungsfindung bei der "Simulation in der Simulation" seine Zielvorstellungen eingeben und die klassischen Planspielentscheidungen offen lassen kann. Somit wird die traditionelle Rollenverteilung der unabhängigen und abhängigen Variablen vertauscht. Das System rechnet ihm die zu treffenden notwendigen Entscheidungen zurück und generiert Erklärungen über die Zusammenhänge (s.u.).

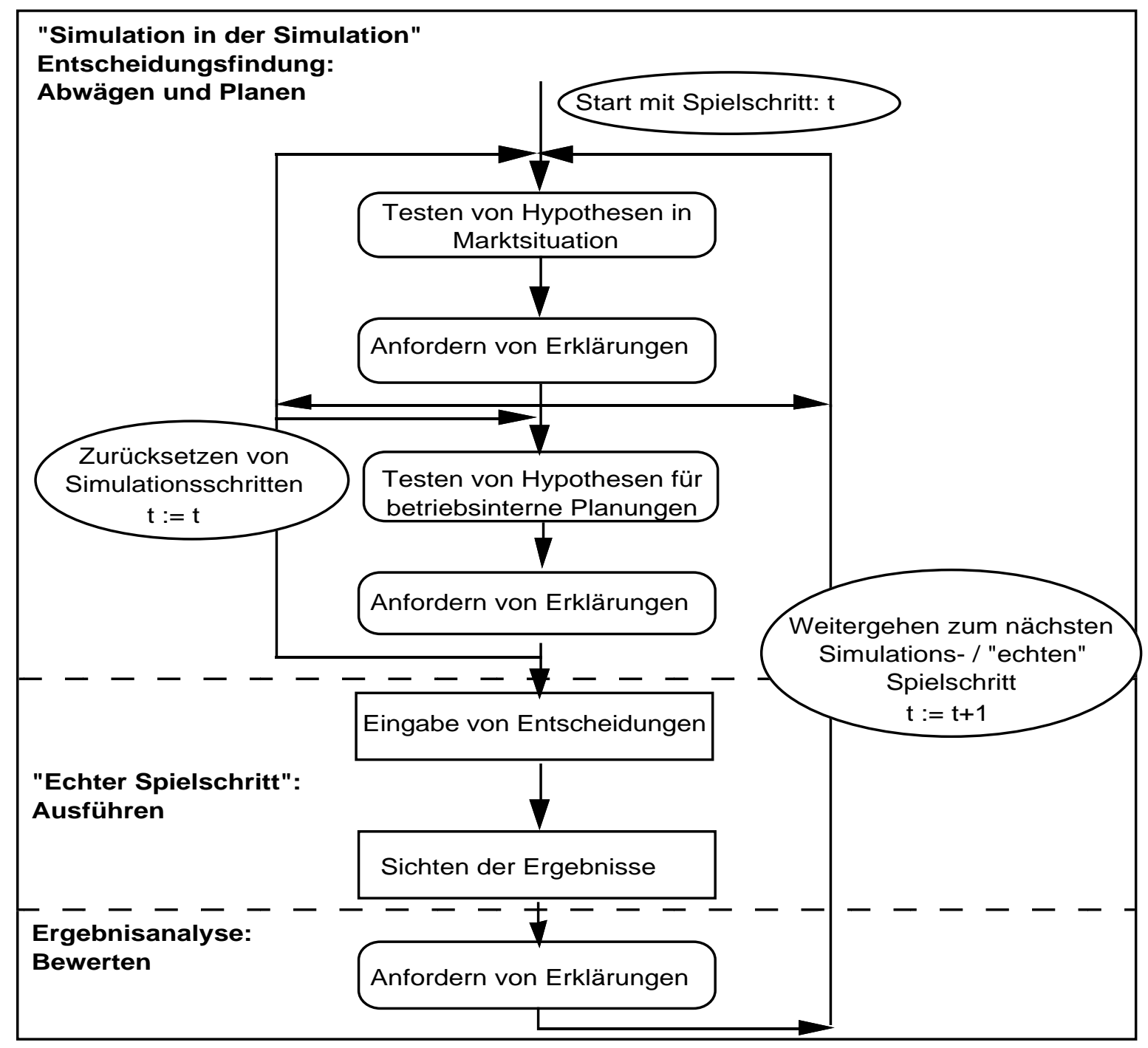

Abb. 1: Ablaufskizze von WULPUS

Eine Handlung "Durchführung eines Spielschrittes in einem Planspiel" zerfällt nach der Rubikontheorie (Heckhausen, 1989; Gollwitzer, 1991) in die vier Handlungsphasen: Abwägen, Planen, Ausführen und Bewerten. In dem prototypischen Planspiel WULPUS werden zusätzlich zur Ausführphase auch die Abwäge-, Plan- und Bewertungsphase unterstüzt. Die Abbildung 1 zeigt eine Ablaufskizze von WULPUS. Der Teil zwischen den beiden gestrichelten Linien enthält die klassischen Planspielanteile, wie sie z.B. auch in LUDUS implementiert sind: Durchführung des "echten" Spielschrittes mit der Eingabe von Entscheidungen und dem Kontrollieren der erzielten Ergebnisse. Der obere Teil zeigt die vom WULPUS-System angebotenen Unterstützungsmöglichkeiten zur Entscheidungsfindung. WULPUS bietet die Hilfe "nur" an, die Kontrolle liegt vollständig beim Benutzer, d.h. das Hypothesentesten und das Anfordern von Erklärungen sind optional (leicht gerundete Ecken). Ein Benutzer hat die Möglichkeit, bis zu vier Spielschritte für die Marktsituation zu durchlaufen, um eine Hypothese 
zur Entwicklung der Marktanteile über einen längeren Zeitraum zu überprüfen (rückführender Pfeil oben rechts). Der Lernende kann das System nach jedem Hypothesen-Spielschritt zurückzusetzen, um Alternativen durchzuprobieren und gegenüberzustellen (rückführender Pfeil oben links). Zu jedem Durchlauf der Marktsimulation kann er Erklärungen anfordern, d.h. er bekommt Hilfe beim Bewerten seiner Planungsergebnisse. Das entspricht einer rekursiven Sichtweise des Handlungsphasenkonzepts (Möbus, 1991).

Nachdem ein Benutzer mit der Marktsituation "zufrieden" ist, kann er aufgrund der dort erreichten Resultate (Werte für Absatz, Preis,...) für sein Unternehmen die betriebsinterne Planung durchführen. Zur Unterstützung der Analyse der Ergebnisse werden dem Benutzer auch an dieser Stelle Erklärungen angeboten. Nach jedem Durchlauf kann er entweder nur die betriebsinterne Planung rückgängig machen, um alternative Lösungen für die innerbetriebliche Planung zu einer Marktsituation zu vergleichen (rückführender Pfeil Mitte links), oder er setzt auch die Marktsimulation auf den Ausgangspunkt zurück und beginnt von neuem. Nachdem die Entscheidungsfindung abgeschlossen ist, werden die Eingaben für den "echten" Spielschritt vorgenommen, hier müssen die klassischen Entscheidungsvariablen eines Planspiels eingegeben werden. Nach der Durchführung des "echten" Spielschrittes kann ein WULPUSSpieler systemgenerierte Erklärungen zur Analyse der Ergebnisse anfordern, falls er sich nicht selbst erklären kann, warum die erreichten Ergebnisse von den intendierten Zielen abweichen.

\subsection{Hypothesentesten in WULPUS}

Der von uns in anderen Domänen entwickelte Hypothesentestansatz (Möbus \& Thole, 1990; Möbus, Thole \& Schröder, 1993) wird mit WULPUS auf die Planspieldomäne übertragen. Dazu wird das Konzept der "Simulation in der Simulation" genutzt: vor dem endgültigen Spielschritt kann ein Spieler Hypothesen über den Zusammenhang von Entscheidungen und Auswirkungen aufstellen und vom System prüfen lassen. Dazu wird die Rollenfestlegung von Entscheidungs- und Ergebnisvariablen aufgelöst: Einzelne Entscheidungen können zu abhängigen und einzelne Ergebnisse zu unabhängigen Variablen werden. Eine Hypothese wird vom WULPUS-Spieler formuliert, indem er Entscheidungen und / oder Ergebnisse nach seinen Wünschen eingibt. Dabei kann er sowohl Entscheidungs- als auch Ergebnisvariablen, deren Werte ihm nicht klar sind, unspezifiziert lassen. Allgemein formuliert lautet die Hypothese: Die eingegebenen Werte sind konsistent. Das System prüft die Hypothese. Dabei können in Abhängigkeit der jeweiligen Variablenbelegung vier Antworttypen auftreten:

1. Die Vorgabe (d.h. der vom Benutzer gesetzte Variablenwert) ist im Hypothesenzusammenhang konsistent.

2. Der Benutzer hatte die Variable unspezifiziert gelassen, und das System hat einen Wert berechnet.

3. Die Vorgabe ist im Hypothesenzusammenhang inkonsistent.

4. Der Benutzer hatte die Variable unspezifiziert gelassen. Das System hatte zu wenig Information, um für sie einen Wert zu berechnen.

Die Rückmeldung von WULPUS auf das Hypothesentesten erfolgt in drei Abstufungen: Zuerst wird dem Benutzer lediglich mitgeteilt, ob seine Hypothese korrekt ist oder nicht. Falls diese Information ausreicht und der Lernende sich das Hypothesentestergebnis selbst erklären kann, braucht er sich die beiden folgenden Systemantworten nicht mehr anzusehen. Falls nötig, erhält der Benutzer im folgenden Schritt auf Anfrage eine Erklärung in Form qualitativer Begründungen. Die letzte Stufe der Systemantworten auf das Hypothesentesten erfolgt quantitativ, d.h. die konkreten Werte werden angezeigt (Abb. 2). 


\section{Vom Benutzer formulierte Hypothese:}

Läßt sich (zum Zeitpunkt t) eine bestimmte Absatzsteigerung bei festgelegtem gesenktem Preis, unveränderten Ausgaben für Werbung, Vertrieb und Forschung erreichen, unter der Annahme, daß die Konkurrenz ihre Absatzstrategie nicht ändert?

\begin{tabular}{|l|l|l|}
\hline Variablen Zeit & \multicolumn{1}{|c|}{ Periode t-1 } & Periode t \\
\hline Absatz & 6.30 Mio. St. & 6.41 Mio. St. \\
Preis & $7.00 \mathrm{DM}$ je St. & $6.50 \mathrm{DM}$ je St. \\
Werbung & 1.7 Mio. DM & 1.7 Mio. DM \\
Vertrieb & 4 Mio. DM & 4 Mio. DM \\
Forschung & 3 Mio. DM & 3 Mio. DM \\
\hline
\end{tabular}

Im Menu ist für die Konkurrenz "keine Veränderungen" ausgewählt.

\section{Systemantworten :}

\section{Stufe (prädikativ):}

Nein, das gesetzte Absatzziel ist unrealistisch.

\section{Stufe (qualitativ):}

Die Tendenz im Marketing-Mix stimmt zwar, ist aber nicht stark genug ausgeprägt.

Ausführliche Erklärung:

Der Absatz läßt sich steigern, wenn der Preis gesenkt wird und die

Ausgaben für Werbung, Vertrieb und Forschung gleich bleiben und außerdem die Konkurrenz ihre Entscheidungen beibehält. Die hier vorgenommene Preissenkung ist aber nicht ausreichend, um das gewünschte Absatzziel zu erreichen.

\section{Stufe (quantitativ):}

Mit den Vorgaben läßt sich ein Absatz von 6.369 Mio. St. erreichen. Die Absatzsteigerung beträgt 1.09 Prozent.

\section{Abb. 2: Skizze einer Hypothese mit WULPUS-Systemantworten}

Die Abbildung 2 zeigt eine Benutzerhypothese über die Realisierbarkeit eines Absatzziels unter den Bedingungen seiner Vorgaben für das Marketing-Mix (Preis, Werbung, Vertrieb und Forschung) und seinen Annahmen über das Mitbewerberverhalten. Sein Ziel ist es, den Absatz von 6.3 auf 6.41 Mio. St. zu steigern. Dabei will er seinen Preis von 7 auf 6.50 DM pro St. senken. Die anderen Variablen des Marketing-Mix sollen unverändert bleiben. Er geht von der Voraussetzung aus, daß die Konkurrenz ihre bisherige Absatzstrategie beibehält. In dem unteren Teil der Abbildung sind die drei Abstufungen der Systemantworten abgebildet. Bei der Formulierung von Hypothesen über die Erreichbarkeit von Absatzzielen bestehen weitere Möglichkeiten:

- Die Variablen des Marketing-Mix können unspezifiziert bleiben. WULPUS berechnet, falls es möglich ist, die mit dem Ziel konsistenten Werte der Variablen.

- Das Absatzziel kann in Form von Strategien w.z.B. Absatzsteigerung formuliert werden.

- Für die Konkurrenz können feste Werte für das Marketing-Mix vorgegeben werden.

- Das Mitbewerberverhalten kann in Form von Strategien angegeben werden. 
Falls in einer Hypothese das Absatzziel als Strategie formuliert wird und der Preis unspezifiziert bleibt, werden die möglichen numerischen Werte bei der dritten Stufe der Systemantwort auf die Hypothese in Form einer Preis/Absatz-Funktion angezeigt. Dabei ist der Bereich, der der Strategie entspricht, gekennzeichnet.

\subsection{Erklärungen in WULPUS}

Die vier unterschiedlichen Fälle des Ausgangs der Hypothesenprüfung werden dem Spieler durch verschiedene "Smileys"-Buttons graphisch auf einfache, verständliche Weise verdeutlicht (Sanderson \& Dougherty, 1993). Im zweiten Fall (unspezifizierte, aber berechenbare Variable) gibt das System einen Vervollständigungsvorschlag für die Variable. Für den dritten Fall (Inkonsistenz) bekommt er auf Wunsch einen Korrekturvorschlag vom System angeboten. Beim vierten Fall (unspezifizierte, nicht berechenbare Variable) nennt WULPUS auf Anfrage die zur weiteren Berechnung der Variablen benötigten Vorgaben, die der Spieler eingeben muß, und macht auf Wunsch zusätzlich einen Eingabevorschlag. In jedem Fall kann der Spieler eine abgestufte Erklärung über das Zustandekommen eines jeden Wertes vom System anfordern. Zum anderen wurde eine Wissensbasis erstellt, die die Vernetzung der betrieblichen Teilbereiche in Form von Regeln enthält. Bei Bedarf kann ein WULPUS-Spieler Erklärungen über das Zustandekommen der resultierenden Ergebnisse aufgrund seiner getroffenen Entscheidungen oder über das Zustandekommen der notwendigen Entscheidungen zur Erreichung seiner Zielvorstellungen vom System anfordern.

Die Erklärungen werden dem Spieler in Abstufungen angeboten, d.h. das Netz der Zusammenhänge der intervenierenden Planspielvariablen wird auf wiederholte Anfrage immer weiter ausgebreitet (Abb. 3). Dabei werden schrittweise Planspielvariablen mit ihren Abhängigkeiten in eine "Unternehmenslandkarte" eingetragen, in der alle betrieblichen Teilbereiche in der Kopfzeile angezeigt werden. Die "Landkarte" dient dem Benutzer zur Orientierung. Die im jeweils letzten Schritt hinzugefügten Konzepte sind fett dargestellt. Die obere Hälfte der Abbildung 3 zeigt die Situation nach dem ersten Schritt: Der Benutzer will sich die Planspielvariable "Endguthaben" im Zusammenhang erklären lassen. Das "Endguthaben" wird im betrieblichen Teilbereich "Finanzstatus" berechnet. Deshalb ist dieser betriebliche Teilbereich geöffnet. Die bedingenden Variablen sind in der "Landkarte" eingetragen. In der unteren Hälfte wurde das Zusammenhangsnetz um zwei weitere Schritte ausgebreitet: Das Netz ist um die bedingenden Variablen der beiden intervenierenden Variablen, "Summe Einzahlungen" und "Summe Auszahlungen", erweitert worden. Die aktuelle Erweiterung des Netzes ist durch fette Knotenlinien markiert. Jeder Variablenknoten enthält zusätzlich die anderen betrieblichen Teilbereiche, in denen die Variable eine Rolle spielt, z.B. kommt "Einzahlungen aus Umsatz" auch im Bereich "ABSATZ / UMSATZ" vor. Weiterhin bekommt der Benutzer Information über den zeitlichen Zusammenhang, d.h. ob Variablen aus der aktuellen Periode "t" oder der Vorperiode "t-1" eingehen. Die Präsentation des Zusammenhangs in Abstufungen hat zwei Gründe: Erstens sollen Selbsterklärungseffekte beim Lernenden gefördert werden, und zweitens ist das gesamte Netz durch die Vielfalt von Zusammenhängen der Teilbereiche unübersichtlich.

Der WULPUS-Benutzer kann sich den mathematischen Zusammenhang der in der "Landkarte" eingetragenen Planspielvariablen anzeigen lassen (Abb. 4). Dabei wird eine Constraint-NetzRepräsentation (Fendler \& Wichlacz, 1987) verwendet, um die unterschiedlichen Berechnungsrichtungen von den Entscheidungen zu den Ergebnissen und von den gewünschten Ergebnissen (Zielen) zu den nötigen Entscheidungen übersichtlich darstellen zu können. 

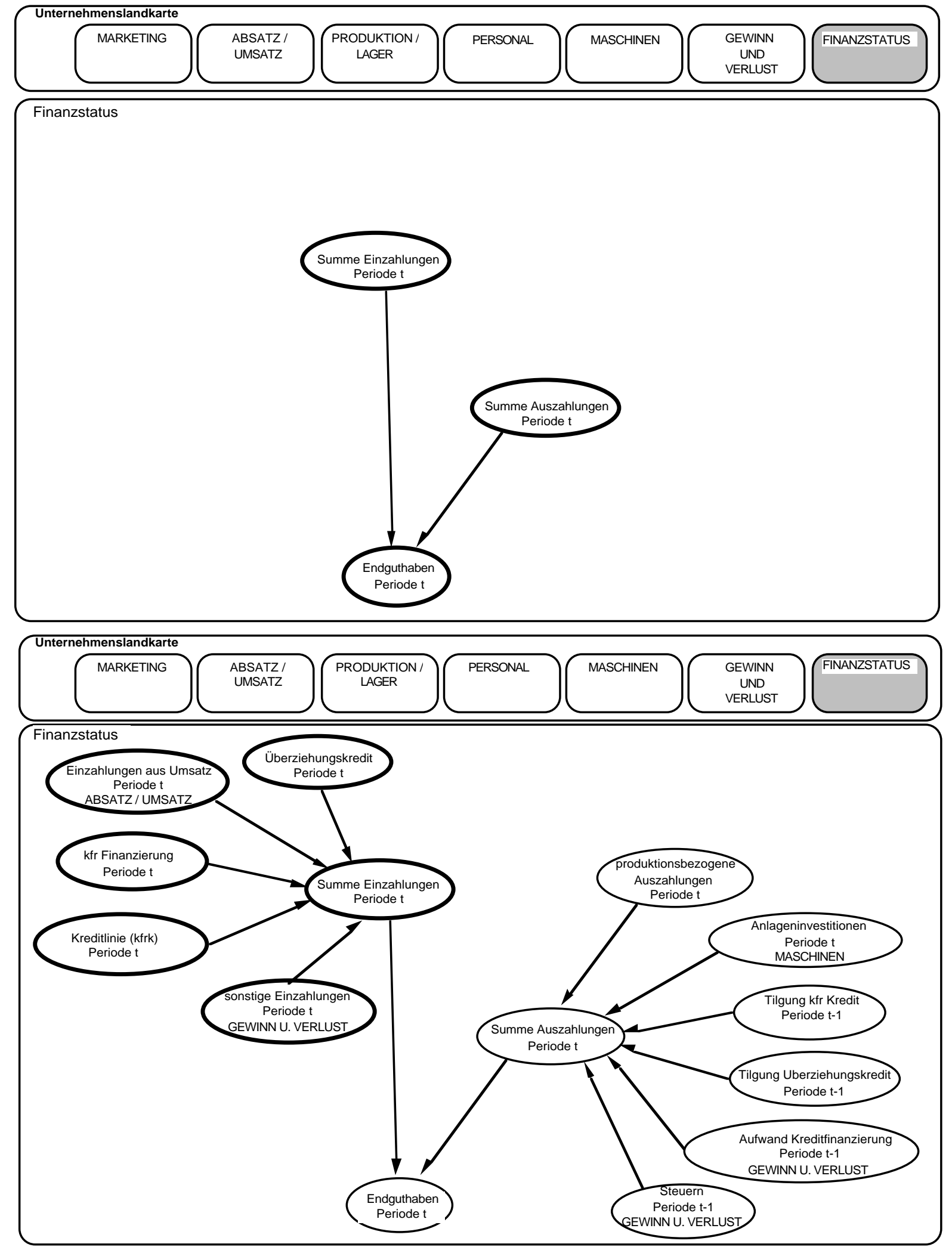

Abb. 3: Darstellung von Abhängigkeiten zwischen den Konzepten in der "Unternehmenslandkarte"

In der Abbildung 4 wird das Beispiel aus der oberen Hälfte der Abbildung 3 wieder aufgegriffen. Immer wenn zwei Werte vorliegen (fetter Rand), kann der dritte (grau) berechnet werden. Der Datenfluß ist durch die Pfeile repräsentiert. Die jeweilige zur Berechnung verwendete Constraint-Gleichung wird dem Benutzer gezeigt. Bei der Darstellung umfangreicher Berechnungen entsteht ein Constraint-Netz. 

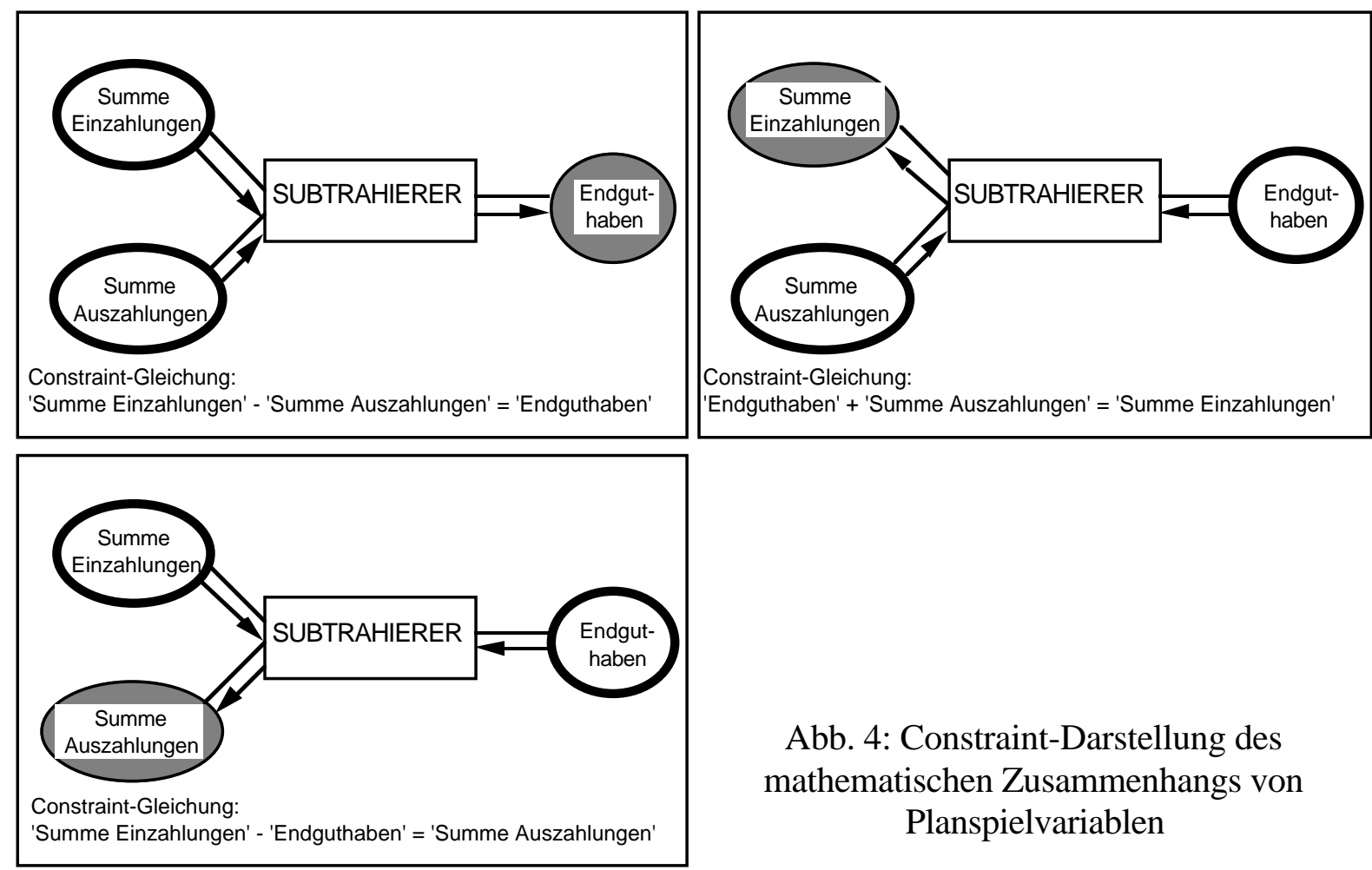
Abb. 4: Constraint-Darstellung des mathematischen Zusammenhangs von Planspielvariablen

WULPUS ist außerdem dazu in Lage, prozentuale Veränderungen von Planspielvariablen im zeitlichen Verlauf zu erklären. Dabei werden die Veränderungen der abhängigen Variablen auf die jeweiligen bedingenden Variablen zurückgeführt. Diese Erklärungen werden beim Bewerten von Ergebnissen als Hilfe angeboten.

\section{Literatur}

EBERT, G., Planspiel: Wettbewerb, Wirtschaftsverlag Bachem, Köln, 1978

ESER, T. W., Planspiel kommunale Wirtschaftsförderung, in H. SPEHL (Hrgs.), Trierer Schriftenreihe zum Schwerpunkt Tourismus, Regional- und Siedlungsentwicklung Nr. 3, Universität Trier, 1992

FENDLER, M. \& WICHLACZ, R., SYCON Symbolische Constraint - Propagierung auf Netzwerken Entwurf und Implementierung, Memo 15, SFB 314: Künstliche Intelligenz - Wissensbasierte Systeme, Leitung: W. WAHLSTER, KI - Labor am Lehrstuhl für Informatik IV, Universität Saarbrücken, 1987

GOLLWITZER, P. M., Abwägen und Planen, Göttingen: Hogrefe, Verlag für Psychologie, 1991

HECKHAUSEN, H., Motivation und Handeln, Berlin: Springer, 1989

MÖBUS, C., Wissenserwerb mit kooperativen Systemen, in: P. GORNY (Hrgs.), Informatik und Schule 1991 - Informatik: Wege zur Vielfalt beim Lehren und Lernen, GI-Fachtagung, Oldenburg, Oktober 1991, Proceedings, Heidelberg: Springer, 1991, 288-298

MÖBUS, C. \& THOLE, H.J., Interactive Support for Planning Visual Programs in the Problem Solving Monitor ABSYNT: Giving Feedback to User Hypotheses on the Basis of a Goals-Means-Relation, in: D.H. NORRIE, H.-W. SIX (eds), Computer Assisted Learning. Proceedings of ICCAL 90, Hagen, F.R.Germany, Lecture Notes in Computer Science, Vol. 438, Heidelberg: Springer, 1990, 36-49

MÖBUS, C., THOLE, H.-J. \& SCHRÖDER, O., Interactive Support of Planning in a Functional, Visual Programming Language, in P. BRNA, S. OHLSSON, H. PAIN (eds), Proceedings AI-ED 93, World Conference on Artificial Intelligence and Education, Edinburgh, 1993, 362 - 369

OCHS, D. \& STEINMANN, B., Planspiel Wirtschaft: Entscheiden und Handeln, Wirtschaftsverlag Bachem, Köln, 1976

RIPPER, J., ZACHAY, A., HERZ, B. \& MERZ, W., Computergestützte Ökonomieplanspiele als Lehr-Lern-Methode: Auswirkungen der Komplexität des Planspiels und des Vorwissens der Benutzer auf den Lernerfolg, in C. MÖBUS (Hrsg.), GI Fachgruppe 1.1.5 / 7.0.1 Intelligente (Tutorielle) Lernsysteme, 6. Arbeitstreffen am 8. / 9. 6. 1993 an der der Carl-von-Ossietzky-Universität Oldenburg, Interne Berichte, Fachbereich Informatik, 1993, 112-121

SANDERSON, D. W. \& DOUGHERTY, D., Smileys - over 650, compiled by David Sanderson, the "Noah Webster of Smileys", O'Reilley \& Associates, Inc., 1993

SCHOEL, D., Erdöl zwischen Markt und Macht, Plan- und Rollenspiele, Wirtsch.-Vlg. Bachem, Köln, 1988

SHUTE, V. J. \& GLASER, R., A Large-Scale Evaluation of an Intelligent Discovery World: Smithtown, Interactive Learning Environments, 1, 1990, 51-77 


\section{Herausgeber}

Friedbert Huber-Wäschle

Peter Widmayer

Institut für Theoretische Informatik

ETH Zentrum

CH-8092 Zürich

Helmut Schauer

Institut für Informatik, Universität Zürich

Winterthurer Strasse 190, CH-8057 Zürich

Die Deutsche Bibliothek - CIP-Einheitsau[nahme

Herausforderungen elnes globalen Informationsverbundes fitir

die Informatik: 25 . GI-Jahrestagung und 13. Schweizer

Informatikertag, Zürich, 18. - 20. September 1995 / GISI '95.

Friedbert Huber-Wäschle ... (Hrsg.). GI. - Berlin ; Heidelberg

; New York ; London ; Paris ; Tokyo ; Hong Kong ; Barcelona

; Budapest : Springer, 1995

(Informatik aktuell)

ISBN 3-540-60213-5

NE: Huber-Wäschle, Friedbert [Hrsg.]; GISI <1995, Zürich〉; Schweizer

Informatikertag <I3, 1995, Zürich>

\section{CR Subject Classification (1995): A.0}

\section{ISBN 3-540-60213-5 Springer-Verlag Berlin Heidelberg New York}

Dieses Werk ist urheberrechtlich geschützt. Die dadurch begründeten Rechte, insbesondere die der Übersetzung, des Nachdrucks, des Vortrags, der Entnahme von Abbildungen und Tabellen, der Funksendung, der Mikroverfilmung oder der Vervielfältigung auf anderen Wegen und der Speicherung in Datenverarbeitungsanlagen, bleiben, auch bei nur auszugsweiser Verwertung, vorbehalten. Eine Vervielfältigung dieses Werkes oder von Teilen dieses Werkes ist auch im Einzelfall nur in den Grenzen der gesetzlichen Bestimmungen des Urheberrechtsgesetzes der Bundesrepublik Deutschland vom 9. September 1965 in der jeweils geltenden Fassung zulässig. Sie ist grundsătzlich vergütungspflichtig. Zuwiderhandlungen unterliegen den Strafbestimmungen des Urheberrechtsgesetzes.

○ Springer-Verlag Berlin Heidelberg 1995

Printed in Germany

Satz: Reproduktionsfertige Vorlage vom Autor/Herausgeber Druck- u. Bindearbeiten: Weihert-Druck GmbH, Darmstadt SPIN: 10484670 33/3140-543210 - Gedruckt auf säurefreiem Papier 


\title{
WULPUS \\ Wissensbasierte Hilfen und Erklärungen für ein betriebswirtschaftliches Planspiel
}

\author{
Claus Möbus, Olaf Schröder, Heinz-Jürgen Thole* \\ Universität Oldenburg, Fachbereich Informatik, \\ Abteilung Lehr- / Lernsysteme, D-26111 Oldenburg, und \\ OFFIS - Oldenburger Forschungs- und Entwicklungsinstitut \\ für Informatik-Werkzeuge und-Systeme, D-26121 Oldenburg \\ \{moebus, schroeder, thole\}@informatik.uni-oldenburg.de
}

\section{Einleitung}

In der betriebswirtschaftlichen Aus- und Weiterbildung werden in zunehmenden Maße Planspiele eingesetzt, um dem steigenden Bildungsbedarf kostengünstig zu begegnen. EDVgestützte Simulationen und Planspiele haben durch die forcierte Vermittlung von Schlüsselqualifikationen eine neue Bedeutung erlangt. Man erhofft sich dadurch einerseits die Vertiefung der Analyse- und Problemlösefähigkeiten und andererseits die Verbesserung der Teamfähigkeit.

Grundlage eines Planspiels bildet ein Modell des Realitätsausschnitts (z.B. Schoel, 1988). Für umfangreiche Problemstellungen, wie z.B. die Führung eines Unternehmens, das sich gegenüber den Mitbewerbern auf den Absatzmärkten zu behaupten hat, muß im Planspiel stärker von der Realität abstrahiert werden (Ebert, 1978; Eser 1992). Dabei bildet die damit verbundene Komplexität des Modells einen Kompromiß zwischen den Extremen:

- große Komplexität, um Realitätsnähe zu garantieren

- geringe Komplexität, um Zusammenhänge transparent werden zu lassen.

Damit Modelle komplexer betriebswirtschaftlicher Gegenstandsbereiche nicht zu stark von der Realität abweichen, weisen sie einen hohen Vernetzungsgrad ihrer Bestandteile auf. Dadurch entsteht zwangsläufig Intransparenz. Der Zusammenhang zwischen Entscheidungs- und Ergebnisvariablen eines Unternehmens wird durch weitere Komponenten wie z.B. das Verhalten der Mitbewerber beeinflußt. In herkömmlichen Planspielen sind die Entscheidungen unabhängige und die Ergebnisse abhängige Variablen. Für den Benutzer ist der Zusammenhang zwischen Entscheidungen und den resultierenden Ergebnissen häufig schwer nachvollziehbar, falls das Domänenwissen des im Planspiel modellierten Weltausschnitts fehlt oder die im Modell enthaltenen Abstraktionen der Realität subjektiv stark verfremdend wirken (Eser, 1992; Ochs \& Steinmann, 1976). Eine weitere Quelle von Schwierigkeiten für den Benutzer ist die Identifikation zeitverzögerter Effekte.

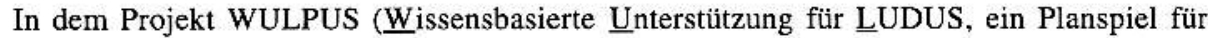
Unternehmensștrategien) wurde ein Ansatz entwickelt, der aufzeigt, wie die Zusammenhänge zwischen verschiedenen betriebswirtschaftlichen Teilbereichen durch einen Hypothesentestansatz (Möbus \& Thole, 1990; Möbus, Thole \& Schröder, 1993) sowie durch wissensbasierte Erklärungen für den Lernenden transparent gemacht werden können. Die Fähigkeit, Hypothesen zu formulieren und zu überprüfen, ist eine Schlüsselqualifikation. Explizites, selbstständiges Formulieren von Hypothesen hat einen effizienzsteigernden und optimierenden Einfluß auf die Wissensakquisition bei den Lernenden (Shute \& Glaser, 1990). Die betriebswirtschaftliche Domäne von WULPUS ist dabei beschränkt auf einen Teilbereich der Inhalte des klassischen Planspiels LUDUS der Firma rado-plan.

* Wir danken Klaus Adam und Hagen von Stuckrad für die Mitwirkung an der Implementation. 
Zentrale Unterscheidungsmerkmale zwischen WULPUS und LUDUS sind:

- Hypothesentesten vor dem "echten Spielschritt": Der Lernende kann sich zum Beispiel die Konsequenzen verschiedener Entscheidungskombinationen berechnen und anzeigen lassen. Im Unterschied zu LUDUS können alternative Entscheidungskombinationen "durchgespielt" werden, sei es, um die Beziehungen zwischen den Variablen zu studieren oder um vor der eigentlichen Entscheidungsfindung Hypothesen über eine günstige Kombination der Entscheidungsvariablen zu formulieren und zu überprüfen.

- Vorwärts- vs. Rückwärtsplanung: Neben der Eingabe von Entscheidungen kann sich der Benutzer Ziele (im Sinne gewünschter Ergebnisse) setzen. Das System versucht dann, diese Hypothese über die Erreichbarkeit der Ziele zu vervollständigen, indem es mit den gewünschten Ergebnissen konsistente Entscheidungskombinationen vorschlägt.

- Korrekturmöglichkeit von inkonsistenten Entscheidungs-_und Ergebnisvariablen: Der Benutzer wird auf nicht erreichbare Ziele hingewiesen und erhält vom System für die betreffenden Variablen Korrekturvorschläge.

- Erklärungskomponente: Der Benutzer erhält wissensbasierte Erklärungen für:

(a) das Zustandekommen der Ergebnisse bei den getroffenen Entscheidungen,

(b) die vom System vorgeschlagenen Entscheidungen zur Erreichung seiner gesetzten Ziele,

(c) die Nichterfüllbarkeit der gesetzten Ziele.

Für die Übertragung des Hypothesentestansatzes auf ein Planspiel sind der Rollentausch der Entscheidungs- und Ergebnisvariablen, das Aufschieben von Berechnungen, bis die benötigten Werte vorliegen, und die Korrekturmöglichkeit von Inkonsistenzen notwendige Voraussetzungen. Da LUDUS diese Einrichtungen nicht bietet, war es nicht möglich, eine Komponente zu entwickeln, die lediglich via Schnittstellen mit LUDUS kommuniziert. Deshalb mußte der Planspielausschnitt vollständig rational rekonstruiert und neu programmiert werden.

\section{Kurzbeschreibung von LUDUS}

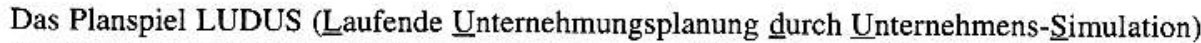
gehört zu der Gruppe der strategischen Konkurrenz-Unternehmensführungsspiele. Es wurde 1990 mit dem deutschen Hochschul-Software-Preis ausgezeichnet. Eingesetzt wird es vornehmlich an Hochschulen, aber auch in Industrie- und Beratungsunternehmen.

In dem Planspiel konkurrieren wahlweise 2 bis 5 Unternehmen mit 1 bis 3 Produkten auf 2 bis 4 Märkten. Die Planungsschwerpunkte liegen im Absatz-, Produktions-, Transport-, Personalund Finanzbereich, wobei die Entscheidungen in jedem dieser betrieblichen Teilbereiche die Auswirkungen der Entscheidungen in den anderen Teilbereichen beeinflussen.

Die Spielteilnehmer von LUDUS müssen ihre Entscheidungen vollständig abgeben. Diese können allerdings in beliebiger Reihenfolge eingegeben werden. Wenn alle Spieler (Unternehmen) ihre Entscheidungsfindung abgeschlossen haben, wird der Spielschritt durchgeführt, d.h. die Ergebnisvariablen werden berechnet. Die Spieler können sich die Auswirkungen ihrer Entscheidungen in Form des Berichtswesens anschauen und ihre Entscheidungen für die neue Periode treffen. Der Benutzer erhält keine Rückmeldung in Form 
von Begründungen über das Zustandekommen der Ergebnisvariablen und hat deshalb u.U. Probleme, seine neuen Entscheidungen den gewünschten Zielen anzupassen.

Als einzige Hilfe zur Entscheidungsfindung stellt LUDUS sogenannte "Planungshilfen" zur Verfügung. Der Spieler hat die Möglichkeit, ein Intervall für den geplanten Absatz und die Produktion anzugeben. Daraufhin berechnet das System z.B. die zum Transport auf weitere Märkte zur Verfügung stehende Menge von Produkten. Der Spieler wird nicht gewarnt, wenn die vorhandene Maschinenkapazität nicht ausreicht, um die geplante Produktion zu realisieren. Außerdem wird die Erreichbarkeit des geplanten Absatzes auf dem jeweiligen Markt vom System nicht geprüft, d.h. das Marktvolumen, die Maßnahmen der konkurrierenden Mitbewerber und das eigene Marketingmix werden nicht berücksichtigt.

LUDUS stellt den Benutzer daher vor folgende Probleme:

1. Es ist nicht deutlich, wie und warum die Ergebnisse aufgrund der getroffenen Entscheidungen zustandegekommen sind (Intransparenz, fehlende Erklärungen).

2. Probehandlungen sind nicht möglich. Entscheidungen können nicht rückgängig gemacht, Alternativen nicht einander gegenübergestellt werden.

3. Man kann sich keine Ziele setzen, und es bleibt unklar, mit welchen Entscheidungen die intendierten Ziele erreichbar sind.

\section{Beschreibung von WULPUS}

Das wissensbasierte Planspiel WULPUS wurde entwickelt, um einen Ansatz aufzuzeigen, der dem Lernenden die Zusammenhänge in komplexen Planspielen transparent macht.

WULPUS bietet dem Benutzer daher die folgenden Möglichkeiten:

1. Erklärungen anzufordern, um die Zusammenhänge zwischen Entscheidungen und Ergebnissen transparent zu machen

2. Probehandlungen durchzuführen und die resultierenden Ergebnisse gegenüberzustellen

3. Ziele zu setzen, also gewünschte Ergebnisse bzw. Teilergebnisse vorzugeben

Der WULPUS-Spieler wird in mehrfacher Hinsicht bei seiner Entscheidungsfindung unterstützt. Dazu wurde das Konzept einer "Simulation in der Simulation" entworfen und realisiert. Der Spieler hat die Möglichkeit, vor dem "echten Spielschritt" Alternativen zu testen, indem er das System wieder zurücksetzt und mit neuen Werten startet. Er kann so mehrere Perioden durchspielen, um zeitlich verzögerte Effekte zu erkennen. Außerdem kann er zusätzlich auf Wunsch die möglichen Mitbewerberentscheidungen variieren und damit das Verhalten der Konkurrenz simulieren, bzw. realistische Hypothesen über deren Verhalten aufstellen und vom System prüfen lassen. WULPUS unterstützt den Planspieler außerdem mit Erklärungen über die Nichterreichbarkeit von Zielen und über den Zusammenhang von Entscheidungs- und Ergebnisvariablen mit Hilfe einer integrierten Expertenwissensbasis. Es hat sich gezeigt, daß Lernende gerade bei komplexen Planspielen oft Probleme haben, Entscheidungen zu treffen, da sie die Auswirkungen nicht überschauen können. Ein Grund dafür ist das fehlende Vorwissen. Daraus folgt, daß Novizen einen geringen Wissenszuwachs beim Arbeiten mit einem komplexen Planspiel haben (vergl. Ripper et al., 1993). 
Sie haben jedoch genaue Vorstellungen über die Ergebnisse, die sie mit den zu treffenden Entscheidungen erreichen wollen. Deshalb haben wir das Planspiel WULPUS so konzipiert, daß ein Spieler zur Entscheidungsfindung bei der "Simulation in der Simulation" seine Zielvorstellungen eingeben und die klassischen Planspielentscheidungen offen lassen kann. Somit wird die traditionelle Rollenverteilung der unabhängigen und abhängigen Variablen vertauscht. Das System rechnet ihm die zu treffenden notwendigen Entscheidungen zurück und generiert Erklärungen über die Zusammenhänge (s.u.).

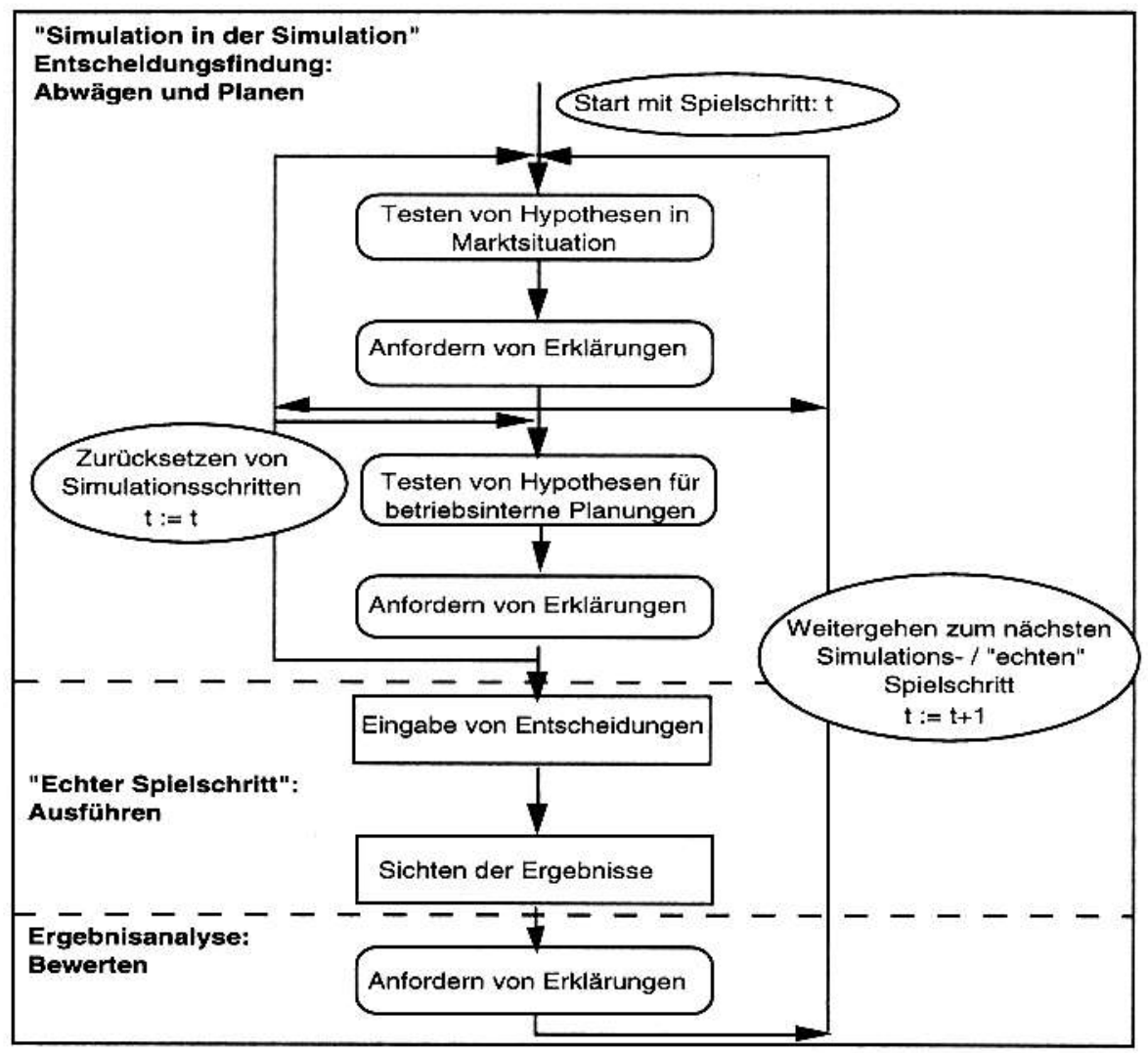

Abb. 1: Ablaufskizze von WULPUS

Eine Handlung "Durchführung eines Spielschrittes in einem Planspiel" zerfällt nach der Rubikontheorie (Heckhausen, 1989; Gollwitzer, 1991) in die vier Handlungsphasen: Abwägen, Planen, Ausführen und Bewerten. In dem prototypischen Planspiel WULPUS werden zusätzlich zur Ausführphase auch die Abwäge-, Plan- und Bewertungsphase unterstüzt. Die Abbildung 1 zeigt eine Ablaufskizze von WULPUS. Der Teil zwischen den beiden gestrichelten Linien enthält die klassischen Planspielanteile, wie sie z.B. auch in LUDUS implementiert sind: Durchführung des "echten" Spielschrittes mit der Eingabe von Entscheidungen und dem Kontrollieren der erzielten Ergebnisse. Der obere Teil zeigt die vom WULPUS-System angebotenen Unterstützungsmöglichkeiten zur Entscheidungsfindung. WULPUS bietet die Hilfe "nur" an, die Kontrolle liegt vollständig beim Benutzer, d.h. das Hypothesentesten und das Anfordern von Erklärungen sind optional (leicht gerundete Ecken). Ein Benutzer hat die Möglichkeit, bis zu vier Spielschritte für die Marktsituation zu durchlaufen, um eine Hypothese 
zur Entwicklung der Marktanteile über einen längeren Zeitraum zu überprüfen (rückführender Pfeil oben rechts). Der Lernende kann das System nach jedem Hypothesen-Spielschritt zurückzusetzen, um Alternativen durchzuprobieren und gegenüberzustellen (rückführender Pfeil oben links). Zu jedem Durchlauf der Marktsimulation kann er Erklärungen anfordern, d.h. er bekommt Hilfe beim Bewerten seiner Planungsergebnisse. Das entspricht einer rekursiven Sichtweise des Handlungsphasenkonzepts (Möbus, 1991).

Nachdem ein Benutzer mit der Marktsituation "zufrieden" ist, kann er aufgrund der dort erreichten Resultate (Werte für Absatz, Preis,...) für sein Unternehmen die betriebsinterne Planung durchführen. Zur Unterstützung der Analyse der Ergebnisse werden dem Benutzer auch an dieser Stelle Erklärungen angeboten. Nach jedem Durchlauf kann er entweder nur die betriebsinterne Planung rückgängig machen, um alternative Lösungen für die innerbetriebliche Planung zu einer Marktsituation zu vergleichen (rückführender Pfeil Mitte links), oder er setzt auch die Marktsimulation auf den Ausgangspunkt zurück und beginnt von neuem. Nachdem die Entscheidungsfindung abgeschlossen ist, werden die Eingaben für den "echten" Spielschritt vorgenommen, hier müssen die klassischen Entscheidungsvariablen eines Planspiels eingegeben werden. Nach der Durchführung des "echten" Spielschrittes kann ein WULPUSSpieler systemgenerierte Erklärungen zur Analyse der Ergebnisse anfordern, falls er sich nicht selbst erklären kann, warum die erreichten Ergebnisse von den intendierten Zielen abweichen.

\subsection{Hypothesentesten in WULPUS}

Der von uns in anderen Domänen entwickelte Hypothesentestansatz (Möbus \& Thole, 1990; Möbus, Thole \& Schröder, 1993) wird mit WULPUS auf die Planspieldomäne übertragen. Dazu wird das Konzept der "Simulation in der Simulation" genutzt: vor dem endgültigen Spielschritt kann ein Spieler Hypothesen über den Zusammenhang von Entscheidungen und Auswirkungen aufstellen und vom System prüfen lassen. Dazu wird die Rollenfestlegung von Entscheidungs- und Ergebnisvariablen aufgelöst: Einzelne Entscheidungen können zu abhängigen und einzelne Ergebnisse zu unabhängigen Variablen werden. Eine Hypothese wird vom WULPUS-Spieler formuliert, indem er Entscheidungen und / oder Ergebnisse nach seinen Wünschen eingibt. Dabei kann er sowohl Entscheidungs- als auch Ergebnisvariablen, deren Werte ihm nicht klar sind, unspezifiziert lassen. Allgemein formuliert lautet die Hypothese: Die eingegebenen Werte sind konsistent. Das System prüft die Hypothese. Dabei können in Abhängigkeit der jeweiligen Variablenbelegung vier Antworttypen auftreten:

1. Die Vorgabe (d.h. der vom Benutzer gesetzte Variablenwert) ist im Hypothesenzusammenhang konsistent.

2. Der Benutzer hatte die Variable unspezifiziert gelassen, und das System hat einen Wert berechnet.

3. Die Vorgabe ist im Hypothesenzusammenhang inkonsistent.

4. Der Benutzer hatte die Variable unspezifiziert gelassen. Das System hatte zu wenig Information, um für sie einen Wert zu berechnen.

Die Rückmeldung von WULPUS auf das Hypothesentesten erfolgt in drei Abstufungen: Zuerst wird dem Benutzer lediglich mitgeteilt, ob seine Hypothese korrekt ist oder nicht. Falls diese Information ausreicht und der Lernende sich das Hypothesentestergebnis selbst erklären kann, braucht er sich die beiden folgenden Systemantworten nicht mehr anzusehen. Falls nötig, erhält der Benutzer im folgenden Schritt auf Anfrage eine Erklärung in Form qualitativer Begründungen. Die letzte Stufe der Systemantworten auf das Hypothesentesten erfolgt quantitativ, d.h. die konkreten Werte werden angezeigt (Abb. 2). 


\section{Vom Benutzer formulierte Hypothese:}

Läß3t sich (zum Zeitpunkt t) eine bestimmte Absatzsteigerung bei festgelegtem gesenktem Preis, unverănderten Ausgaben für Werbung, Vertrieb und Forschung erreichen, unter der Annahme, daß die Konkurrenz ihre Absatzstrategie nicht ändert?

\begin{tabular}{|l|l|l|}
\hline Variablen Zeit & \multicolumn{1}{|c|}{ Periode t-1 } & \multicolumn{1}{|c|}{ Periode t } \\
\hline Absatz & $6.30 \mathrm{Mio}$. St. & 6.41 Mio. St. \\
Preis & 7.00 DM je St. & 6.50 DM je St. \\
Werbung & 1.7 Mio. DM & 1.7 Mio. DM \\
Vertrieb & 4 Mio. DM & 4 Mio. DM \\
Forschung & 3 Mio. DM & 3 Mio. DM \\
\hline
\end{tabular}

Im Menu ist für die Konkurrenz "keine Veränderungen" ausgewăhlt.

\section{Systemantworten :}

\section{Stufe (prädikativ):}

Nein, das gesetzte Absatzziel ist unrealistisch.

\section{Stufe (qualitativ):}

Die Tendenz im Marketing-Mix stimmt zwar, ist aber nicht stark genug ausgeprägt.

\section{Ausführliche Erklärung:}

Der Absatz läßt sich steigern, wenn der Preis gesenkt wird und die

Ausgaben für Werbung, Vertrieb und Forschung gleich bleiben und außerdem die Konkurrenz ihre Entscheidungen beibehält. Die hier vorgenommene Preissenkung ist aber nicht ausreichend, um das gewünschte Absatzziel zu erreichen.

\section{Stufe (quantitativ):}

Mit den Vorgaben läßt sich ein Absatz von 6.369 Mio. St. erreichen. Die

Absatzsteigerung beträgt 1.09 Prozent.

\section{Abb. 2: Skizze einer Hypothese mit WULPUS-Systemantworten}

Die Abbildung 2 zeigt eine Benutzerhypothese über die Realisierbarkeit eines Absatzziels unter den Bedingungen seiner Vorgaben für das Marketing-Mix (Preis, Werbung, Vertrieb und Forschung) und seinen Annahmen über das Mitbewerberverhalten. Sein Ziel ist es, den Absatz von 6.3 auf 6.41 Mio. St. zu steigern. Dabei will er seinen Preis von 7 auf $6.50 \mathrm{DM}$ pro St. senken. Die anderen Variablen des Marketing-Mix sollen unverändert bleiben. Er geht von der Voraussetzung aus, daß die Konkurrenz ihre bisherige Absatzstrategie beibehält. In dem unteren Teil der Abbildung sind die drei Abstufungen der Systemantworten abgebildet. Bei der Formulierung von Hypothesen über die Erreichbarkeit von Absatzzielen bestehen weitere Möglichkeiten:

- Die Variablen des Marketing-Mix können unspezifiziert bleiben. WULPUS berechnet, falls es möglich ist, die mit dem Ziel konsistenten Werte der Variablen.

- Das Absatzziel kann in Form von Strategien w.z.B. Absatzsteigerung formuliert werden.

- Für die Konkurrenz können feste Werte für das Marketing-Mix vorgegeben werden.

- Das Mitbewerberverhalten kann in Form von Strategien angegeben werden. 
Falls in einer Hypothese das Absatzziel als Strategie formuliert wird und der Preis unspezifiziert bleibt, werden die möglichen numerischen Werte bei der dritten Stufe der Systemantwort auf die Hypothese in Form einer Preis/Absatz-Funktion angezeigt. Dabei ist der Bereich, der der Strategie entspricht, gekennzeichnet.

\subsection{Erklärungen in WULPUS}

Die vier unterschiedlichen Fälle des Ausgangs der Hypothesenprüfung werden dem Spieler durch verschiedene "Smileys"-Buttons graphisch auf einfache, verständliche Weise verdeutlicht (Sanderson \& Dougherty, 1993). Im zweiten Fall (unspezifizierte, aber berechenbare Variable) gibt das System einen Vervollständigungsvorschlag für die Variable. Für den dritten Fall (Inkonsistenz) bekommt er auf Wunsch einen Korrekturvorschlag vom System angeboten. Beim vierten Fall (unspezifizierte, nicht berechenbare Variable) nennt WULPUS auf Anfrage die zur weiteren Berechnung der Variablen benötigten Vorgaben, die der Spieler eingeben muß, und macht auf Wunsch zusätzlich einen Eingabevorschlag. In jedem Fall kann der Spieler eine abgestufte Erklärung über das Zustandekommen eines jeden Wertes vom System anfordern. Zum anderen wurde eine Wissensbasis erstellt, die die Vernetzung der betrieblichen Teilbereiche in Form von Regeln enthält. Bei Bedarf kann ein WULPUS-Spieler Erklärungen über das Zustandekommen der resultierenden Ergebnisse aufgrund seiner getroffenen Entscheidungen oder über das Zustandekommen der notwendigen Entscheidungen zur Erreichung seiner Zielvorstellungen vom System anfordern.

Die Erklärungen werden dem Spieler in Abstufungen angeboten, d.h. das Netz der Zusammenhänge der intervenierenden Planspielvariablen wird auf wiederholte Anfrage immer weiter ausgebreitet (Abb. 3). Dabei werden schrittweise Planspielvariablen mit ihren Abhängigkeiten in eine "Unternehmenslandkarte" eingetragen, in der alle betrieblichen Teilbereiche in der Kopfzeile angezeigt werden. Die "Landkarte" dient dem Benutzer zur Orientierung. Die im jeweils letzten Schritt hinzugefügten Konzepte sind fett dargestellt. Die obere Hälfte der Abbildung 3 zeigt die Situation nach dem ersten Schritt: Der Benutzer will sich die Planspielvariable "Endguthaben" im Zusammenhang erklären lassen. Das "Endguthaben" wird im betrieblichen Teilbereich "Finanzstatus" berechnet. Deshalb ist dieser betriebliche Teilbereich geöffnet. Die bedingenden Variablen sind in der "Landkarte" eingetragen. In der unteren Hälfte wurde das Zusammenhangsnetz um zwei weitere Schritte ausgebreitet: Das Netz ist um die bedingenden Variablen der beiden intervenierenden Variablen, "Summe Einzahlungen" und "Summe Auszahlungen", erweitert worden. Die aktuelle Erweiterung des Netzes ist durch fette Knotenlinien markiert. Jeder Variablenknoten enthält zusätzlich die anderen betrieblichen Teilbereiche, in denen die Variable eine Rolle spielt, z.B. kommt "Einzahlungen aus Umsatz" auch im Bereich "ABSATZ/ UMSATZ" vor. Weiterhin bekommt der Benutzer Information über den zeitlichen Zusammenhang, d.h. ob Variablen aus der aktuellen Periode " $t$ " oder der Vorperiode " $t-1$ " eingehen. Die Präsentation des Zusammenhangs in Abstufungen hat zwei Gründe: Erstens sollen Selbsterklärungseffekte beim Lernenden gefördert werden, und zweitens ist das gesamte Netz durch die Vielfalt von Zusammenhängen der Teilbereiche unübersichtlich.

Der WULPUS-Benutzer kann sich den mathematischen Zusammenhang der in der "Landkarte" eingetragenen Planspielvariablen anzeigen lassen (Abb. 4). Dabei wird eine Constraint-NetzRepräsentation (Fendler \& Wichlacz, 1987) verwendet, um die unterschiedlichen Berechnungsrichtungen von den Entscheidungen zu den Ergebnissen und von den gewünschten Ergebnissen (Zielen) zu den nötigen Entscheidungen übersichtlich darstellen zu können. 

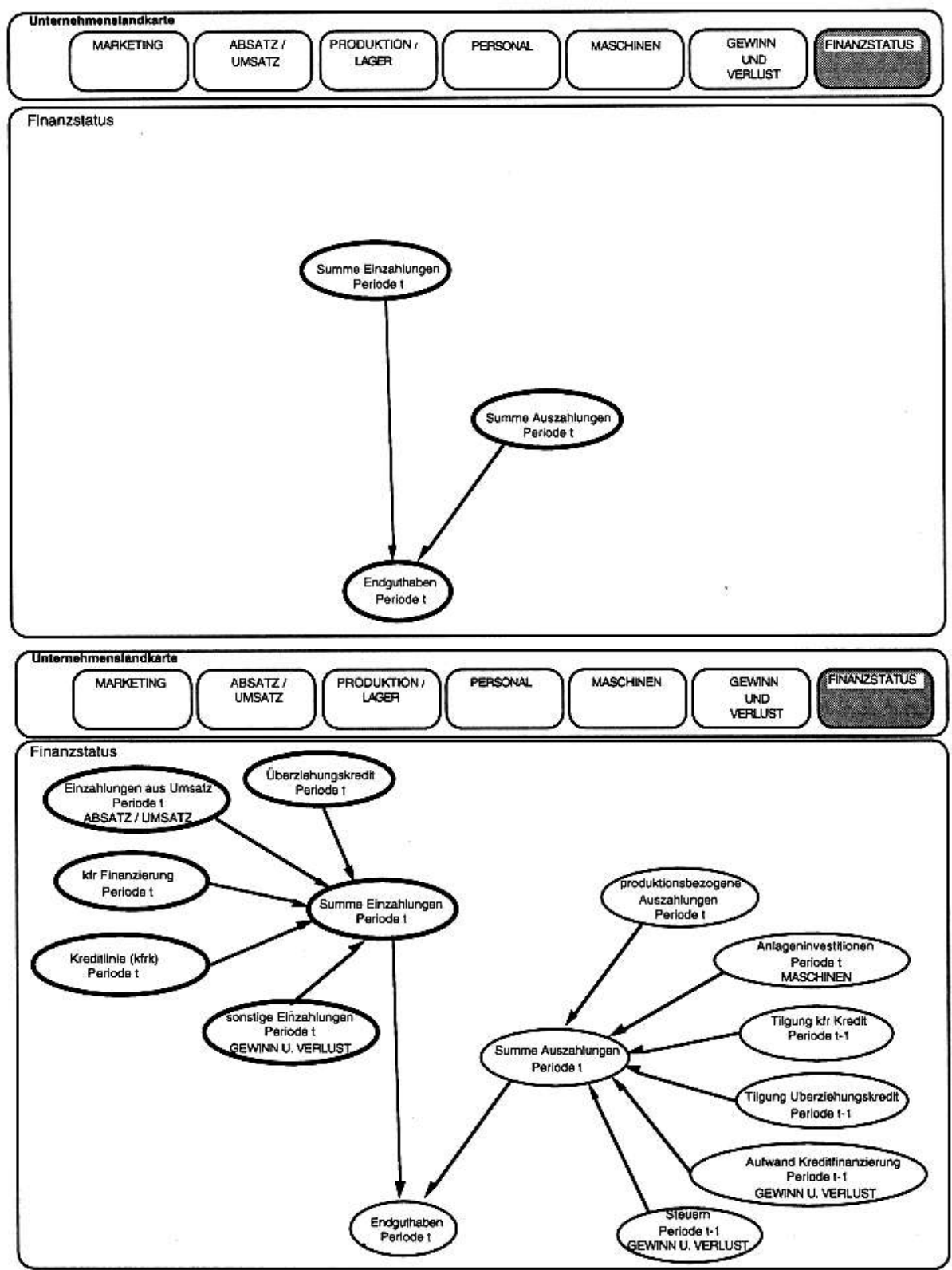

Abb. 3: Darstellung von Abhängigkeiten zwischen den Konzepten in der "Unternehmenslandkarte"

In der Abbildung 4 wird das Beispiel aus der oberen Hälfte der Abbildung 3 wieder aufgegriffen. Immer wenn zwei Werte vorliegen (fetter Rand), kann der dritte (grau) berechnet werden. Der Datenfluß ist durch die Pfeile repräsentiert. Die jeweilige zur Berechnung verwendete Constraint-Gleichung wird dem Benutzer gezeigt. Bei der Darstellung umfangreicher Berechnungen entsteht ein Constraint-Netz. 

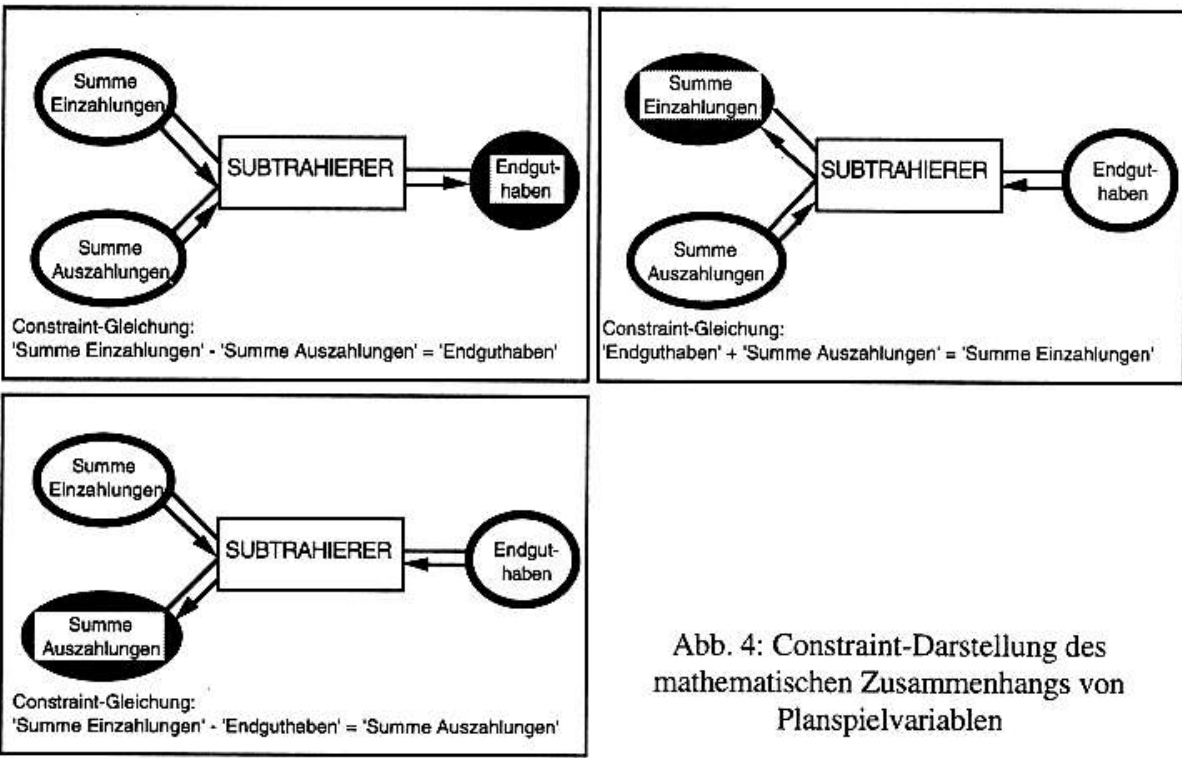

Abb. 4: Constraint-Darstellung des mathematischen Zusammenhangs von Planspielvariablen

WULPUS ist außerdem dazu in Lage, prozentuale Veränderungen von Planspielvariablen im zeitlichen Verlauf zu erklären. Dabei werden die Veränderungen der abhängigen Variablen auf die jeweiligen bedingenden Variablen zurückgeführt. Diese Erklärungen werden beim Bewerten von Ergebnissen als Hilfe angeboten.

\section{Literatur}

EBERT, G., Planspiel: Wettbewerb, Wirtschaftsverlag Bachem, Köln, 1978

ESER, T. W., Planspiel kommunale Wirtschaftsförderung, in H. SPEHL (Hrgs.), Trierer Schriftenreihe zum Schwerpunkt Tourismus, Regional- und Siedlungsentwicklung Nr. 3, Universität Trier, 1992

FENDLER, M. \& WICHLACZ, R., SYCON Symbolische Constraint - Propagierung auf Netzwerken Entwurf und Implementierung, Memo 15, SFB 314: Künstliche Intelligenz - Wissensbasierte Systeme, Leitung: W. WAHLSTER, KI - Labor am Lehrstuhl für Informatik IV, Universität Saarbrücken, 1987

GOLLWITZER, P. M., Abwägen und Planen, Göttingen: Hogrefe, Verlag für Psychologie, 1991

HECKHAUSEN, H., Motivation und Handeln, Berlin: Springer, 1989

MÖBUS, C., Wissenserwerb mit kooperativen Systemen, in: P. GORNY (Hrgs.), Informatik und Schule 1991 - Informatik: Wege zur Vielfalt beim Lehren und Lernen, GI-Fachtagung, Oldenburg, Oktober 1991, Proceedings, Heidelberg: Springer, 1991, 288-298

MÖBUS, C. \& THOLE, H.J., Interactive Support for Planning Visual Programs in the Problem Solving Monitor ABSYNT: Giving Feedback to User Hypotheses on the Basis of a Goals-Means-Relation, in: D.H. NORRIE, H.-W. SIX (eds), Computer Assisted Learning. Proceedings of ICCAL 90, Hagen, F.R.Germany, Lecture Notes in Computer Science, Vol. 438, Heidelberg: Springer, 1990, 36-49

MÖBUS, C., THOLE, H.-J. \& SCHRÖDER, O., Interactive Support of Planning in a Functional, Visual Programming Language, in P. BRNA, S. OHLSSON, H. PAIN (eds), Proceedings AI-ED 93, World Conference on Artificial Intelligence and Education, Edinburgh, 1993, 362 - 369

OCHS, D. \& STEINMANN, B., Planspiel Wirtschaft: Entscheiden und Handeln, Wirtschaftsverlag Bachem, Köln, 1976

RIPPER, J., ZACHAY, A., HERZ, B. \& MERZ, W., Computergestützte Ökonomieplanspiele als Lehr-Lem-Methode: Auswirkungen der Komplexität des Planspiels und des Vorwissens der Benutzer auf den Lernerfolg, in C. MÖBUS (Hrsg.), GI Fachgruppe 1.1.5/7.0.1 Intelligente (Tutorielle) Lernsysteme, 6. Arbeitstreffen am 8. / 9. 6. 1993 an der der Carl-von-Ossietzky-Universität Oldenburg, Interne Berichte, Fachbereich Informatik, 1993, 112-121

SANDERSON, D. W. \& DOUGHERTY, D., Smileys - over 650 , compiled by David Sanderson, the "Noah Webster of Smileys", O'Reilley \& Associates, Inc., 1993

SCHOEL, D., Erdöl zwischen Markt und Macht, Plan- und Rollenspiele, Wirtsch.-Vlg. Bachem, Köln, 1988 SHUTE, V. J. \& GLASER, R., A Large-Scale Evaluation of an Intelligent Discovery World: Smithtown, Interactive Learning Environments, 1, 1990, 51-77 